\title{
La integración de la evaluación de políticas públicas en el proceso presupuestario
}

\section{Eduardo Zapico Goñi *}

\section{Introducción}

En los últimos años estamos viviendo en Europa un importante impulso de la evaluación de políticas públicas. Nuevos programas universitarios, revistas especializadas, asociaciones profesionales, etc..., se han presentado y se están desarrollando con rapidez, en torno a este tema. El esfuerzo en sí mismo es válido y meritorio. A medio y largo plazo puede tener consecuencias importantes en el funcionamiento de la Administración. Muy posiblemente se puedan identificar iniciativas de evaluación de programas y/o políticas públicas que estén teniendo resultados inmediatos. Más importancia tiene el efecto general que estos esfuerzos van a tener en desarrollar la capacidad de oferta y fomentar una nueva cultura de evaluación que impulse la propia demanda de análisis de los programas públicos.

No obstante, en este artículo se plantea y analiza la importancia y posibilidad que la integración de la evaluación en el proceso presupuestario pueda tener no sólo para consolidar su demanda sino principalmente para recoger el fruto directo del esfuerzo invertido: reducción de costes, mejora en la productividad, mayor calidad de los servicios, eficacia e impacto final de los programas públicos e incluso la mejor formulación y desarrollo de los programas públicos.

La evaluación de programas no deja de ser un riesgo para Hacienda pues permite al gestor argumentar a favor de la necesidad de un aumento del gasto. No obstante, también es una oportunidad para identificar recortes de gasto sostenibles y que no afecten a la calidad de los servicios públicos y a la eficacia o impacto final de los programas públicos.
La evaluación de programas no proporciona una solución inmediata contra la indisciplina presupuestaria, y mucho menos a nivel macro. Pero puede orientar a Hacienda en el diseño del marco de referencia para imponer una mayor disciplina de gasto (fijación de techos de gasto, distribución de límites agregados entre prioridades sectoriales, etc...). La integración de la evaluación al proceso presupuestario, a nivel de centro gestor, puede ser útil para que éstos se adapten a una situación en la que se necesitan reasignar recursos dentro de un límite presupuestario impuesto desde Hacienda en el marco de un programa de estabilidad.

El proceso presupuestario es a su vez una oportunidad para el desarrollo de la evaluación de políticas publicas. Pero a cualquier estudiante de ciencias políticas o funcionario con una cierta experiencia en la gestión del gasto público no se le escapa la idea de que este proceso representa también un desafío importante. El presupuesto se puede percibir metafóricamente como una moneda con una cara en la que se refleja su aspecto técnico y racional y con una cruz en la que se refleja su aspecto negociado.

En principio, nadie se opone a la idea de utilizar la evaluación de programas o políticas públicas a la hora de redistribuir los recursos disponibles. Han sido múltiples los estudios realizados desde los años 60 en favor de introducir la técnica de presupuesto por programas o los sistemas integrados de presupuestación (D. Novick, 1969; A. SHICK; 1978, etc..). El objetivo de las reformas presupuestarias basadas en estos modelos no era otro sino el de reasignar recursos en base al análisis de los resultados de la gestión de los programas presupuestarios. Aunque estos modelos de reforma se han abandonado, en muchos países se siguen propo- 
niendo modelos muy parecidos con términos casi sinónimos: i.e. el presupuesto por resultados o la integración de la medición de resultados en la resignación de recursos (PUMA, 1997, p. 36).

Sin embargo, son escasas las experiencias conocidas en el mundo en las que este ejercicio de "racionalidad presupuestaria" se realice. ¿Por qué ocurre esto? ¿Hasta qué punto es así? ¿En qué medida es erróneo el modelo teórico de referencia? En las próximas secciones se analizan algunas líneas de respuesta a estas preguntas con el fin de replantear la discusión sobre las posibilidades de integrar la evaluación de programas y presupuesto. Al final del artículo se analizan las condiciones necesarias y se proponen algunas iniciativas para proporcionar una mayor coherencia al proceso presupuestario.

\section{La integración del presupuesto y la evaluación de resultados en la práctica}

En la práctica, el enlace de la evaluación de programas y el proceso presupuestario ha resultado ser más difícil de aplicar de lo que se esperaba. Las evidencias empíricas recogidas sobre las experiencias de varios gobiernos (US, UK, Canadá, Alemania, España, Suecia y Finlandia) indican que incluso en Suecia, donde se ha reconocido la necesidad y se han hecho importantes esfuerzos de integrar de manera regular y sistemática la evaluación de resultados en la elaboración del presupuesto, su aplicación ha sido débil e infrecuente (GRAY, JeNKINS y SEGSWORTH, 1993,- p. 191). Otros estudios en países nórdicos han corroborado estos resultados (P. OvReiID, 1997, p. 9).

A nivel de gobiernos regionales o subcentrales los resultados son parecidos. Los resultados de un estudio sistemático realizado por la General Accounting Office (Oficina de Auditoría del Congreso) y la Congressional Budget Office (Oficina de Presupuesto del Congreso) en los EE.UU. sobre el mismo tema ofrecen unos resultados similares. Tras analizar el proceso presupuestario en los cinco Estados que habían realizado un mayor esfuerzo y que se consideraban más avanzados en la introducción de técnicas presupuestarias se demostró y reconoció por las Administraciones implicadas que existía muy pocas evidencias de que los resultados de la gestión influyeran en la asignación de los recursos públicos durante el proceso presupuestario. En general, se reconoce que en esencia el presupuesto en estos Estados se sigue realizando de manera incrementalista.

Pero estos resultados no significan que los esfuerzos realizados en las Administraciones más avanzadas no hayan tenido ninguna recompensa. En el proceso presupuestario de algunos países (Suecia, RU, los Países Bajos) se dispone ahora de más información y mucho más relevante que hace unos pocos años sobre niveles de economía y eficiencia en la gestión. Hay una cierta conexión entre la eficiencia operativa y la asignación de recursos. Esto es especialmente cierto para créditos presupuestarios que financian el funcionamiento operativo de los servicios. No obstante, la relación entre el seguimiento de la gestión y la asignación de recursos está pendiente de realizarse (OCDE, 1993, p. 11).

En el RU y en Suecia el Tesoro y el Ministerio de Finanzas respectivamente exigen a los centros gestores la presentación de indicadores de resultados al solicitar nuevos créditos de un año para otro. El nivel de estos indicadores se va gradualmente desarrollando. No obstante, los centros de gasto normalmente no están dispuestos a que se les evalúe el resultado de sus programas (Gray, JeNKINS y Segsworth, 1993, pp. 49, 50). Las dificultades de la implantación del presupuesto por resultados y otras reformas de la gestión pública han sido subestimadas (T. VerheIjen y D. COOMBES, 1998, p. 394).

A pesar de estos débiles resultados, la evaluación se sigue percibiendo hoy como de gran utilidad para promover una nueva cultura de gestión presupuestaria centrada en el resultado e impacto de los programas más que en la formalidad de los procedimientos y en el ahorro inmediato. De los comentarios del comisario encargado de Control Financiero de la Comisión Europea se deduce que la evaluación se percibe como un buen instrumento para facilitar el tránsito de una cultura de gasto, donde gobernar o gestionar bien se confunde con aumentar los créditos presupuestarios, a una cultura de resultados, donde el directivo público se centra y es responsable por el grado de eficienciă y eficacia alcanzado al ejecutar su presupuesto (A. GRADIN, 1996, p. 27).

Si bien hay consenso sobre los beneficios que puede aportar la evaluación de programas, hemos visto antes que hasta ahora han sido escasas las experiencias conocidas en el mundo en las que se haya ejercido la aracionalidad presupuestaria de manera sistemática. ¿Por qué ocurre esto? ¿En qué medida es erróneo el modelo teórico de referencia? 


\section{Análisis crítico de la teoría en la que se apoya un sistema integrado de gestión presupuestaria}

\subsection{Limitaciones para su implantación}

Se suelen mencionar razones como la falta de liderazgo político, la no voluntad de ser medido y evaluado por parte de los gestores, la falta de apoyo de los funcionarios de presupuesto y de los profesionales de cada sector o política, etc... para explicar la dificultad de introducir la evaluación de programas en el proceso presupuestario.

Aunque muchas de estas razones tienen su peso, existen otras razones de importancia que tradicionalmente no se han percibido o considerado con la suficiente atención por los responsables de la reforma presupuestaria. Uno de los factores que empiezan ahora a plantearse en Suecia (NAB) como fundamentales para integrar con éxito la evaluación y el presupuesto es el que se utilicen indicadores que sean de relevancia y de interés para los miembros del Parlamento.

Pero lo importante es reconocer y aceptar que incluso si se identifican los indicadores relevantes para los miembros del Parlamento, éstos no serían sino unos criterios de éxito útiles para orientar el debate presupuestario. Es ingenuo esperar que la evaluación sustituya a la negociación política. El proceso de elaboración del presupuesto a alto nivel es un proceso de negociación en el que se enfrentan intereses contrapuestos. El presupuesto es negociación y conflicto (A. WIDAVSKY, 1979, p. 4) y seguirá siéndolo. La confrontación de intereses no es el reflejo de un mal funcionamiento del proceso presupuestario, sino una característica de la realidad en la que se desarrolla el proceso de elaboración del presupuesto: los recursos son escasos en relación a las necesidades humanas, por naturaleza siempre insatisfechas.

Esto no debe de entenderse como una conclusión pesimista o derrotista como tradicionalmente se ha percibido desde la Administración o desde el mundo de la economía (STOCKMAN, 1984) sino como un desafío contextual con el que tienen que enfrentarse el Gobierno y la Administración al asignar y gestionar el gasto. El problema no está en la existencia del conflicto presupuestario sino en la manera de confrontarlo. En algunas Administraciones hay una tendencia a evitar el conflicto. Éste se oculta y se le deja diluir en el tiempo, se decide no tomar nin- guna decisión de reasignación de recursos, por entenderse que no habría capacidad para gestionar una posible cadena de conflictos y agravios comparativos. Pero el conflicto puede confrontarse, se puede aprovechar la oportunidad que ofrece la crisis para aclarar y si es posible mejorar la situación para todos (ZAPICO, 1993), las crisis son oportunidades para ejercitar la creatividad.

Gran parte del camino para la reforma del presupuesto se habrá recorrido cuando los responsables de diseñarla y llevarla a cabo acepten que el estilo de decisión de políticas públicas, 0 . el comportamiento y valores de decisión de los participantes en el proceso de decisión son tan importantes o más que las propias técnicas de presupuestación. Esto no es una propuesta o conclusión derrotista, la evaluación de políticas puede ayudar y considerar como objetivo propio el fomentar una cultura de gasto $o$ un estilo de decisión presupuestaria que se adecúe al contexto económico financiero.

Tambiér a nivel técnico el proceso presupuestario refleja las luchas de poder internas dentro de la Administración. La tradicional desconfianża entre Hacienda y el gestor es otra de las razones que pueden explicar la escasa influencia de las técnicas de medición de resultados y evaluación en el proceso presupuestario. Al gestor le resulta sospechoso escuchar las reformas presupuestarias que se han propuesto desde organismos internacionales basadas en la idea de reasignar recursos desde actividades menos eficientes a las más eficientes (P. OvRELID, 1997, p. 7).

Aunque la retórica es atractiva, en la practica es tradicional que Hacienda no premia sino que de hecho penaliza al gestor que por eficiencia o por otra causa consigue que le sobren unos recursos al final del ejercicio (Erkki LIIKANEn, 1996, p. 35). En principio el gestor desconfía que siendo eficiente vaya a recibir más recursos en época de restricción presupuestaria.

De nuevo, el seguimiento de la gestión y la evaluación de los programas públicos no pueden entenderse ni pueden aplicarse de manera mecánica en el proceso presupuestario. $\mathrm{Ni} \mathrm{a}$ nivel político ni a nivel técnico. Es necesario modificar el sistemas de incentivos que realmente motivan al gestor público. La reforma de los formatos y técnicas presupuestarias y la introducción de la evaluación de programas va detrás o a continuación de los cambios en el comportamiento o estilo de decisión en la asignación y utilización de los recursos y no al revés. Éste fue uno de los motivos por los que se fracasó en la implantación de la reforma presupuestaria.

\subsection{Incoherencia entre modelo de gestion empresarial y la gestión presupuestaria en el sector público}

El modelo de gestión integrada del presupuesto se basa en la teoría tradicional de la gestión empresarial. La idea de aplicar 
los resultados de la evaluación de programas en la resignación de recursos tradicionalmente suele ir unida a la teoría racional de decisión. A mayor productividad o. eficiencia de una actividad mayores recursos se le deberían asignar en el ejercicio siguiente. El modelo se orienta a la maximización de beneficios o utilidad esperada y se basa en definitiva en el principio de competencia entre los actores presupuestarios que se supone estarían más motivados para mejorar los resultados de la gestión.

Esta teoría tiene una cierta utilidad también demostrada para el sector público. De hecho, se está utilizando en algunos países (RU, Países Bajos, NZ, etc..), en algunos servicios públicos (salud, Policía) o incluso en los servicios centrales de la Administración de manera limitada, i.e. gastos de funcionamiento. Los valores competitivos tienen mucha importancia en la motivación de comportamientos productivos tanto en la empresa privada como en la Administración.

Por supuesto, el funcionamiento apropiado del modelo competitivo exige una mayor autonomía de trabajo del gestor público y una capacidad de seguimiento y evaluación de resultados de la gestión. El departamento de presupuestos sólo puede conceder mayor autonomía de gasto en la medida que las unidades que reciben más poder y responsabilidad de gasto tengan capacidad de informar y demostrar los buenos resultados de su gestión financiera (N. FLYNN y F. STREHL (eds.), 1996, p. 264).

Sin embargo, hasta ahora, el impacto de la llamada Nueva Gestión Pública al intentar aumentar la eficiencia en la gestión del gasto público ha sido relativamente débil (T. VeRHEIJEN y D. COOMBES, 1998, pp. 391 y ss.).

Cada vez se acepta más la necesidad de tener que analizar el contexto y adaptar el modelo de presupuesto a las características del mismo. Sin embargo es un hăndicap en este sentido la poca capacidad que existe en la Administración para aprender de las iniciativas llevadas a cabo (Otsen y Peters, 1996, p. 14). Hasta mediados los 90 ningún país había evaluado en profundidad el impacto en la eficiencia y eficacia de la Administración como consecuencia de las recientes reformas presupuestarias. Hasta ahora estas evaluaciones han sido ad boc e inadecuadas en la mayoría de los países debido principalmente a problemas de medición y la sensibilidad política del tema (OCDE, 1993, p. 9). "Los programas de reforma de la gestión pública normalmente no disponen de procedimientos de retroalimentación, evaluación y corrección o, disponen de ellos, suelen ser despreciados y no se utilizan» (VerheIJen y COOMBES, 1998, p. 394).

Algunos países han dado importantes pasos en esta línea. Australia ha realizado una evaluación comprehensiva de sus iniciativas de reforma en el sector público (Australia, Task Force on Management Improvement, 1992). Un análisis sobre seguimiento de resultados realizado por Sylvie Trosa (MAYNE y ZAPICO (eds.), 1997, cap. 4) se basa en una investigación del impacto de las reformas realizadas en el Reino Unido (UK, Cabinet Office, 1994). En Canadá la Oficina del Controlador General (Tesoro y el Auditor General (Parlamento) han evaluado el resultado de reformas llevadas a cabo para el desarrollo de la evaluación de programas (MAYNE y ZAPICO (eds.), 1997, cap. 2).

Una de las condiciones necesarias en un contexto presupuestario para que tenga éxito la reforma clásica del sistema integrado de presupuestación es que haya una cierta estabilidad de recursos" (OCDE, 1993, p. 5). Este tipo de dificultades ya se habían anticipado en investigaciones académicas (HOFSTEDE, 1981, p. 193). Aunque el modelo tradicional de reforma presupuestaria es útil en un contexto de estabilidad y certidumbre, puede ser inapropiado o insuficiente para el tipo de trabajo que se realiza en muchas organizaciones públicas, especialmente en los departamentos centrales y en las organizaciones de profe sionales y en general en la red de organizaciones que participa en el proceso presupuestario, bajo gran complejidad y en una realidad "multifacética” (J. OLSEn y PETERS, 1996, p: 14).

Las reformas centradas en la eficiencia y en la competición por los recursos han tenido un resultado limitado a la consecución de un recorte del gasto relativamente reducido. Dado el contexto de incertidumbre e inestabilidad, gran parte de los recursos públicos se están gestionando de manera eficiente pero están resolviendo problemas que han dejado de existir o se están proporcionando servicios que la sociedad no valora de la misma manera que años atrás. Esto es más fácil que ocurra en aquellas reformas presupuestarias enfocadas en la mejora de la productividad y que dan por supuesta la continuidad de las políticas públicas, sus objetivos y sus marcos institucionales de trabajo.

La evaluación de la gestión del gasto no debería sólo plantearse cuestiones del tipo single loop: ¿qué tal lo estamos haciendo?, ¿en qué medida estamos trabajando bien?, etc..., sino además plantearse también las de tipo: double loop: ¿tiene sentido que hagamos esta tarea? (ARGYRIS según referencia en F. LeEUw, 1996, p. 74). La integración de la evaluación en el proceso presupuestario puede proporcionar grandes resultados en términos de reducción del déficit en la medida en que incorpore valoraciones relacionadas con el segundo orden de aprendizaje al que se refiere las cuestiones del tipo double loop.

Por supuesto, la evaluación de programas ha evolucionado, está más orientada a responder las preguntas del tipo double loop y por lo tanto puede ser de utilidad para mejorar el proceso presupuestario. El valor añadido de la Evaluación de Programas reside en el propio proceso de evaluación más que en el informe final de sus resultados.

El problema sigue siendo cómo acercar la acción evaluadora y los evaluadores a la acción de presupuestar y los decisores responsables de la asignación de recursos y. viceversa. A conti- 
nuación se exponen algunas condiciones e iniciativas para llevar a cabo ese acercamiento.

\section{Condiciones para mejorar la coherencia interna de un sistema presupuestario}

Históricamente ha existido una diferenciación entre el presupuesto, la auditoría y la evaluación que no ha ido acompañada de un esfuerzo correspondiente de coordinación. Hoy el presupuesto y la evaluación son actividades separadas, realizadas por personal con distinta preparación, valores, intereses y criterios de éxito. Para identificar posibles mejoras en su integración o coherencia es necesario realizar un buen diagnóstico de la situación actual.

Aunque en cáda Administración el diagnóstico tendrá sus características particulares, siempre resulta interesante conocer los rasgos generales de experiencias comparadas en otros países. Siguiendo el estudio comparado mencionado en el apartado segundo, la falta de integración se puede analizar desde cuatro puntos de vista complementarios, según el tipo de integración.

Por ejemplo, de manera intuitiva se suele valorar primero el grado de integración desde una perspectiva funcional. La integración funcional puede medirse por el grado de utilidad o consistencia del resultado de una actividad para con la otra y viceversa. Como ya se ha dicho, el presupuesto y la evaluación son funciones muy distintas con productos diferentes que en realidad reflejan racionalidades legítimamente diferentes. La cuestión no es meramente técnica. Es de esperar que su integración requiera un esfuerzo continuo de coordinación que aceptando la pluralidad de perspectivas tienda a aproximarlas.

La mayoría de los casos analizados en este estudio no presentan evidencias de que exista coherencia funcional-entre el presupuesto y la evaluación de políticas publicas. La asignación de recursos presupuestarios está movida por una convicción doctrinal (ideología de mercado) más que por un esfuerzo de concepción o evaluación de las políticas públicas.

Pero la falta de integración puede analizarse también desde otros puntos de vista. Por ejemplo, la integración organizacional o grado de cohesión de las estructuras y procesos en los que se lleva a cabo el presupuesto y la evaluación lógicamente también influye. La claridad y coherencia en el diseño de roles y responsa- bilidades de los actores que componen la red de organizaciones que participan en el ciclo presupuestario, entendido de manera amplia, es un aspecto fundamental en este sentido.

Pues bien, el estudio dirigido por GraY y otros vuelve a mostrar que la distribución de competencias e interrelaciones entre los participantes en el proceso presupuestario no está bien definida en ninguno de los países analizados. Aunque sí se reconoce que el presupuesto y la auditoría están relativamente mejor integrados en algunos países (Alemania, España, Francia).

Otro tipo de integración a considerar es la aintegración informativa" o grado en el que se comparten sistemas de información y comunicación, de acceso común o con bases de datos coherentes, compatibles, etc... De nuevo la integración es muy limitada o inexistente. Aunque en los últimos años se han realizado avances considerables respecto a sistemas de medición de resultados e información para la toma de decisiones, no hay evidencias claras de que existan marcos o canales comunes que faciliten la comunicación entre la evaluación y el presupuesto. En Suecia los sistemas de información que se utilizan en el proceso presupuestario se usan también para la evaluación de las políticas públicas.

Por último y quizás una de las más importantes sea la perspectiva socio-organizacional. La integración social puede entenderse como la homogeneidad en la cultura, preparación, experiencia y conocimiento del personal responsable de realizar estas funciones.

Existe una preparación similar en el personal de presupuesto y auditoría en Alemania y en España, dada la tradición legal y la formación similar que reciben. Pero la evaluación queda fuera de estas consideraciones. La integración de la evaluación y el presupuesto exige la existencia de una cultura profesional y administrativa común o al menos semejante, de manera que se abra espacio suficiente para la cooperación entre las distintas comunidades de expertos. En ningún país se dan las condiciones adecuadas para garantizar una comunicación eficaz entre ambas comunidades, según el estudio mencionado, de GRAY y otros. Sin embargo, en Suecia se han realizado programas de formación conjunta entre evaluadores y responsables del presupuesto. En este país se ha dado la aparente paradoja de que la propia institucionalización de la evaluación parece haber establecido barreras para su apertura a otras funciones tradicionalmente enraizadas en la Administración como el presupuesto.

En base a este diagnóstico de la desintegración de la evaluación y el presupuesto en algunos de los países occidentales se presenta a continuación una serie de medidas que pueden ayudar a superar esta situación. Estas medidas no pretenden ser una lista cerrada e infalible para conseguir integrar presupuesto y evaluación. Se trata más bien de una serie de condiciones, necesarias no suficientes, que hay que cumplir y obstáculos a su- 
perar según cada situacion. Está claro que existe una interacción importante entre algunas de las propuestas que deberá tenerse en cuenta al diseñar el marco de reforma.

a) Condiciones técnicas:

- Rediseño de la estructura, procesos y funciones de evaluación y presupuesto, estableciendo una división clara de responsabilidades. Este reparto de roles debe ser consensuado y acorde con las capacidades y recursos disponibles por cada centro participante.

- Desarrollo de los sistemas de información y canales de comunicación entre los centros responsables de la evaluación y presupuesto. Estos sistemas y canales deben garantizar una interacción eficaz. El intercambio de información y la explotación de las bases de datos comunes o compartidas necesita disfrutar de unos niveles aceptables de fiabilidad y credibilidad.

- Formación de una masa crítica de evaluadores y expertos en presupuesto, distribuida de manera homogénea entre los centros gestores y centros de presupuesto y evaluación, con capacidad de entendimiento mutuo (aceptar interdependencias, identificar valores comunes, etc...).

- Introducción de incentivos y/o eliminación de desincentivos. Aunque sólo algunos países lo vienen practicando, principalmente los nórdicos (P. OvReLID, 1997, p. 6), es evidente que para que la integracion tenga éxito el beneficio potencial que ofrece la evaluación debería ser al menos compartido por los centros gestores y Hacienda. Y por la misma razón habría que evitar o reducir los efectos negativos de la negociación en las disponibilidades de crédito presupuestario (p.e., evitar la devolución automática al Tesoro de todos los ahorros conseguidos con la evaluación, ya sea por recortes identificados o aumentos de productividad y eficacia, etc..., concediendo una mayor discrecionalidad en su uso.

\section{b) Condiciones político-institucionales:}

- Adaptación del estilo de decisión en la asignación de recursos. Modificación de las reglas de la negociación presupuestaria en el Parlamento. Fomento de una nueva cultura político-administrativa en el tratamiento de conflictos presupuestarios (ZAPICO, 1995).

- Formulación de acuerdos institucionales con mayoría parlamentaria sostenible y adopción de la legislación apropiada para crear un marco políticoadministrativo como inicio de un cambio de actitu- des respecto a la evaluación de políticas públicas. Así se ha hecho ya, por ejemplo, en Suecia y.Francia. Además, se ha demostrado que la evaluación tiende a estar más integrada cuando ésta se realiza por órganos supremos e independientes de auditoría como ocurre en el RU (Gray, Jenkins y SegsWORTH, 1993; DERLIEN, 1990).

\section{Conclusiones}

Se ha analizado la importancia y posibilidades de integrar la evaluación en el proceso presupuestario. Sin embargo, se ha observado cómo hasta ahora las experiencias conocidas, de Administraciones de reconocida importancia, en las que se ha intentado dicha integración, su resultado no ha sido positivo. El enlace de la evaluación de programas y el proceso presupuestario ha resultado ser más difícil de aplicar de lo que se esperaba.

No obstante, a pesar de los débiles resultados hasta ahora conseguidos, se han presentado evidencias claras de que la evaluación se sigue percibiendo como de gran utilidad para el presupuesto y la gestión financiera. Ésta puede facilitar el tránsito de una cultura de gasto, donde gobernar o gestionar bien se confunde con aumentar los créditos presupuestarios, a una cultura de resultados, donde el directivo público se centra y es responsable por el grado de eficiencia y eficacia alcanzado al ejecutar su presupuesto. Su integración en el proceso presupuestario puede proporcionar importantes resultados en términos de reducción relevantes de costes, de manera sostenible, si incorpora valoraciones y proporciona información relacionadas con el segundo orden de aprendizaje para evitar que gran parte de los recursos públicos se orienten a resolver problemas que han dejado de existir o se presten servicios que la sociedad ya no necesita.

Pero esta integración de la evalución y el presupuesto no puede entenderse como un mecanismo para imponer unos criterios técnicos supuestamente objetivos y neutros en la asignación de recursos. La evaluación puede aspirar a ser un factor importante pero no el único a tener en cuenta en el proceso presupuestario. La utilidad de la evaluación se verá potenciada en el proceso presupuestario en la medida en que Hacienda y el Gobierno desarrollen su capacidad de confrontar con eficacia los conflictos que surgen al identificar prioridades y reasignar recursos. La evaluación de programas y las técnicas de decisión presupuestaria deben intentar ir más allá del tradicional criterio marginalista recogido en la clásica pregunta: ¿A qué programa debería de asignarse la última peseta disponible, para maximizar la eficiencia de los recursos? La integración de la evaluación en el proceso presupuestario puede proporcionar grandes re- 
sultados en términos de reducción del déficit en la medida en que incorpore como criterios de éxito la adaptación y el aaprendizajes en el diseño y desarrollo de los programas presupuestarios.

El verdadero valor añadido de la Evaluación de Programas reside en el propio proceso de evaluación más que sus resultados. La expectativa de asignar recursos estrictamente de acuerdo a los resultados de la evaluación de las políticas públicas es ingenua (OCDE). La evaluación de programas no puede entenderse ni debe aplicarse de manera mecánica en el proceso presupuestario. Gran parte del beneficio de una buena evaluación debe haberse materializado antes de formularse el informe final. El proceso de evaluación podría y debería orientarse a la consolidación de los canales de comunicación e interacción entre decisores, participantes y grupos de interés (stake-bolders) en general del programa presupuestario en cuestión. Esto aumen- taría la coherencia interna del proceso presupuestario ya que permitiría reducir la incertidumbre ainnecesaria. o evitable, que se suele plantear en el proceso de asignación de los recursos cuando no se gestionan de manera apropiada las interdependencias (WILDAVSKY y ZAPICO, 1993).

Finalmente, se ha destacado la importancia de un diagnóstico de la situación actual para proponer mejoras en la coherencia o integración del proceso presupuestario. Como método de diagnóstico se ha propuesto el análisis del déficit de integración desde cuatro puntos de vista complementarios, según el tipo de integración: Organizacional, funcional, informativa y social. En base al diagnóstico realizado en estudios comparados se han propuesto una serie de líneas generales para iniciar un proceso de acercamiento entre la evaluación y el presupuesto.

\section{Bibliografia}

GRADIN, A. (1996), Introduction. En Acbieving Better Value from European Community Funds, FEE, Bruselas, p. 27.

Gray, A.; Jenkins, B. y Segsworth, B. (1993), Budgeting, Auditing and Evaluation. Transaction P. New Brunswick (USA).

Hofstede, G. (1981), "A Management Control of Public and Not For Profit Activities". Accounting Organizations and Society, 6 (3): pp. 193-211.

Landau, M. y Stout, R. Jr. (1979), *A to Manage is Not to Control: Or the Folly of Type II Errorsn. Public Administration Review, March/April, p. 151.

LIIKANEN, E. (1996), amproving Financial Management in the EU. En Acbieving better value from European Community Funds, FEE, Belgium, p. 35.

MAYNE, J. y ZAPICO, E. (1997), Monitoring Performance in the Public Sector. Transaction P. New Brunswick (USA).

MetCalFe, L. y RuCHaRDS, S. (1990), Improving Public Management. $2 .^{\text {id }}$ edition, Sage, pp. $210,96,40$ y ss.

_(1993), •A Public Management: From Imitation to Implementation. En J. KOOIMAN (ed.), Modern Government, Sage, pp. 173-189.

Novick, D. (1969), :The Origen and History of Programe Budgeting. En @®Programe Budgeting@@ Halt, Rinehart.

OLSEN, J. y PETERS, G. (1996), Lessons from Experience: Experiential learning in Administrative Reform in Eight countries' Scandinavian. University Press, Oslo.
OVRELID, P. (1997), -The impact of linking evaluation with Budgeting, Directorate of Public management in Norway. Paper presented at the Conference on Evaluation in Oslo, EES, p. 9.

PUMA (1997), Modern Budgeting. OCDE, París, p. 36.

Stockman (1984), El Triunfo de la Politica sobre el Presupuesto. OMB, President of the US.

SHCK, A. (1978), the Road to PPB. En A. HYDE y J. SHAFrIT, Govermment Budgeting, Moore PC.

VerheiJen, T. y COOMBES, D. (eds.) (1998), sInnovations. En Public Management, Edward Elgar, Cheltenham, RU, pp. 391 y ss.

WILDAVSKY, A. y ZAPICO, E. (eds.) (1993), National Budgeting for Economic and Monetary Union. Martinus Nijhoff P., London, pp. 119 y ss.

WILDAVsKY, A. (1975), Budgeting: A Comparative Theory of Budgetary Processes. Little Brown \& Co., pp. 9, 163, 4 .

(1988), A Cultural Theory of Budgeting. International Journal of Public Administration, 11 (6): pp. 651-677.

WINHAM, G. (1977), •A Negotiations as a Management Process. World Politics, 30 (1): pp. $87-114,97$.

ZAPICO, E. (1995), Proceso Presupuestario como un proceso de negociaciones complejas. En Cuadernos de Relaciones Laborales, UCM, Madrid. 
